\title{
Induction of ventricular arrhythmias by programmed ventricular stimulation: a prospective study on the effects of stimulation current on arrhythmia induction
}

\author{
PETER L WEISSBERG, ARCHER BROUGHTON, RICHARD W HARPER, \\ ALISON YOUNG, AUBREY PITT
}

From the Cardiology Service, Alfred Hospital and Baker Medical Research Institute, Melbourne, Australia

SUMMARY A protocol for programmed ventricular stimulation is described in which the effect of increasing stimulation current on ventricular refractoriness and arrhythmia induction was specifically examined. The protocol was evaluated prospectively in 70 patients undergoing electrophysiological study for documented or suspected ventricular arrhythmias. Programmed electrical stimulation was performed at the right ventricular apex and outflow tract using single and double extrastimuli and burst pacing. Stimulation currents of 2, 5, 10, and $20 \mathrm{~mA}$ were used in ascending order. The initial (lowest) current was never less than twice diastolic threshold and was maintained during each stimulation run until refractoriness was reached. The current was then increased to the next level to facilitate premature capture until refractoriness was encountered at $20 \mathrm{~mA}$ or a sustained arrhythmia occurred.

Ventricular arrhythmias were induced in 34 patients, 31 of whom had presented with a sustained ventricular arrhythmia. The incidence of induced arrhythmias was low in those patients who had presented with symptoms alone, a non-sustained arrhythmia, or a sustained arrhythmia in association with a predisposing clinical event. Only one patient with a negative result had further ventricular arrhythmias during the mean follow up period of 15 months. Although each increase in stimulation current caused a decrease in measured ventricular refractoriness, this resulted in only four arrhythmias. Only one arrhythmia was induced above $5 \mathrm{~mA}$.

These results suggest that this simple protocol using two extrastimuli and a single stimulation current of $5 \mathrm{~mA}$ will reliably identify most patients who have symptomatic ventricular arrhythmias.

The use of programmed ventricular stimulation has been advocated to identify patients at risk of recurrent ventricular arrhythmias. The ability to induce arrhythmias by this technique is determined by both the number of extrastimuli introduced and the current used. ${ }^{1}$ While aggressive protocols (using multiple premature stimuli and high current) maximise

Requests for reprints to Dr Peter L Weissberg, Department of Cardiovascular Medicine, East Birmingham Hospital, Birmingham B95ST.

Accepted for publication 23 June 1987 the chance of inducing an arrhythmia in a susceptible patient, they may also induce a number of "artefactual" arrhythmias of dubious clinical importance. ${ }^{2}$ Consequently, there is little agreement on what is the most appropriate stimulation protocol for clinical use, particularly with respect to the optimal stimulation current. ${ }^{3-5}$ While the ideal protocol should be sufficiently sensitive to identify those at risk and sufficiently specific to exclude those who are not, it should also be simple enough to be acceptable to both patients and physicians.

In this study we have prospectively evaluated a stimulation protocol which uses a maximum of two 
Table 1 Details of the 70 patients in the study

\begin{tabular}{llll}
\hline Group & & & \\
\hline $1(n=34)$ & $2(n=11)$ & $3(n=5)$ & $4(n=20)$ \\
\hline $\begin{array}{l}\text { Reason for referral } \\
\text { Documented } \\
\text { sustained VT }\end{array}$ & $\begin{array}{l}\text { Documented } \\
\text { VF }\end{array}$ & $\begin{array}{l}\text { Documented } \\
\text { non-sustained }\end{array}$ & $\begin{array}{l}\text { Syncope and/or } \\
\text { VTmptoms }\end{array}$ \\
$\begin{array}{l}\text { Diagnosis } \\
\text { CAD 23 }\end{array}$ & CAD 8 & CAD 2 & CAD 6 \\
$\begin{array}{l}\text { VHD 1 } \\
\text { CM 2 } \\
\text { NSHD 8 }\end{array}$ & NSHD 3 & $\begin{array}{l}\text { VHD 1 } \\
\text { NSHD 2 }\end{array}$ & $\begin{array}{l}\text { CHD 2 } \\
\text { NSHD 12 }\end{array}$ \\
$\begin{array}{c}\text { Positive result } \\
28(76 \%)\end{array}$ & $5(45 \%)$ & $1(20 \%)$ & $2(10 \%)$ \\
\hline
\end{tabular}

CAD, coronary heart disease; CHD, congenital heart disease; CM, cardiomyopathy; NSHD, no structural heart disease; VF, ventricular fibrillation; VHD, valvar heart disease; VT, ventricular tachycardia.

extrastimuli and we have specifically examined the effect of stimulation current on ventricular refractoriness and arrhythmia induction. The protocol was assessed in 70 consecutive patients suspected of having ventricular arrhythmias.

\section{Patients and methods}

\section{PATIENTS}

We prospectively studied 70 ( 50 men and 20 women; mean (range) age 55 (16-73)) consecutive patients referred to the cardiology service at the Alfred Hospital for investigation of documented or suspected ventricular arrhythmias (table 1 ). The patients were divided into four groups on the basis of their presentation: group 1, 34 patients in whom sustained ventricular tachycardia had been documented; group 2, 11 patients who had presented with documented ventricular fibrillation; group 3, five patients in whom non-sustained ventricular tachycardia had been documented; and group 4, 20 patients with either unexplained syncope or symptoms suggestive of tachycardia but no documented ventricular arrhythmia. In 16 patients the original clinical arrhythmia had occurred during a likely precipitating event or within one month of acute infarction (eight in group 1 , seven in group 2, and one in group $4)$. In the remaining 54 patients no precipitating event could be identified. The underlying diagnosis was coronary artery disease in 39 (35 of whom had suffered a previous myocardial infarction), valvar or congenital heart disease in four, and cardiomyopathy in two. In the remaining 25 patients there was no clinical evidence of structural heart disease. All antiarrhythmic drugs were stopped at least four half lives before ventricular stimulation. All patients gave informed consent.

\section{STIMULATION PROTOCOL}

The patients were fasted and sedated with oral diazepam $(10 \mathrm{mg})$. Two 7F pacing electrodes were introduced by percutaneous puncture under $1 \%$ prilocaine anaesthesia. A tripolar catheter was advanced from the right femoral vein across the tricuspid ring to record the His bundle electrogram. A quadripolar electrode inserted into the right antecubital vein was positioned first to pace the right ventricular apex at 100,120 , and $150 / \mathrm{min}$, then to pace the right ventricular outflow tract at $150 / \mathrm{min}$. A single extrastimulus was used to scan diastole at each pacing rate. The procedure was then repeated using double extrastimuli before burst pacing was carried out at both sites at pacing rates $170-240 / \mathrm{min}$. Pulse duration was fixed at $2 \mathrm{~ms}$ throughout the study.

A programmable stimulator (Medtronic 5325) was used to deliver four levels of stimulation current: 2 , $5,10,20 \mathrm{~mA}$. The initial current was set at $2 \mathrm{~mA}$ for patients with a threshold $<1 \mathrm{~mA}$ and $5 \mathrm{~mA}$ if the threshold was $>1 \mathrm{~mA}$. Since no patient had a diastolic threshold $>2.5 \mathrm{~mA}$ this ensured that the initial current was never less than twice the diastolic threshold. When coupled to a $500 \Omega$ test impedance the current delivered was within $5 \%$ of each nominal value. The coupling interval between the last of eight paced beats and the first extrastimulus was reduced in $20 \mathrm{~ms}$ steps from $380-300 \mathrm{~ms}$ and then in $10 \mathrm{~ms}$ steps until refractoriness was reached at the initial current. The current was then increased to the next level to facilitate premature capture and so on until refractoriness was eventually reached at $20 \mathrm{~mA}$. The current was then returned to the initial level and diastole was scanned with a single extrastimulus at each pacing rate before a second extrastimulus was introduced. The coupling interval between the first and second extrastimulus was similarly decreased from $300 \mathrm{~ms}$ after the coupling interval between the last paced beat and the first extrastimulus had been set at $30 \mathrm{~ms}$ longer than the effective refractory period measured at the initial current. During burst pacing, current was only increased above the initial current if this was required to sustain ventricular capture.

Ventricular tachycardia was categorised as either: sustained; lasting $>30 \mathrm{~s}$ or requiring immediate DC cardioversion; non-sustained lasting $>5$ beats but $<30$ s; uniform, stable QRS configuration; multiform, unstable QRS configuration. All subjects completed the protocol unless a sustained arrhythmia was induced in which case no attempt was made to repeat arrhythmia induction at another stimulation current. As non-sustained multiform tachycardia was never induced as the sole arrhythmia, we did not analyse its occurrence in patients in whom sustained arrhythmias were induced. A patient was judged to 
Table 2 Details of patients in whom ventricular arrhythmias were induced

\begin{tabular}{|c|c|c|c|c|c|c|}
\hline $\begin{array}{l}\text { Patient } \\
\text { No }\end{array}$ & $C A D$ & $\begin{array}{l}\text { Reason for } \\
\text { for study }\end{array}$ & $\begin{array}{l}I P C \\
(m A)\end{array}$ & $\begin{array}{l}\text { VA } \\
\text { induced }\end{array}$ & $\begin{array}{l}\text { Site and mode } \\
\text { of induction }\end{array}$ & $\begin{array}{l}\text { Current } \\
\text { (mA) }\end{array}$ \\
\hline $\begin{array}{r}1 \\
2 \\
3 \\
4 \\
5 \\
6 \\
7 \\
8 \\
9 \\
10 \\
11 \\
12 \\
13 \\
14 \\
15 \\
16 \\
17 \\
18 \\
19 \\
20 \\
21 \\
22 \\
23 \\
24 \\
25 \\
26 \\
27 \\
28 \\
29 \\
30 \\
31 \\
32 \\
33 \\
34\end{array}$ & $\begin{array}{l}+ \\
+ \\
+ \\
+ \\
0 \\
0 \\
+ \\
0 \\
+ \\
+ \\
+ \\
+ \\
0 \\
0 \\
0 \\
+ \\
0 \\
+ \\
+ \\
+ \\
+ \\
+ \\
+ \\
0 \\
+ \\
+ \\
+ \\
0 \\
0 \\
+ \\
+ \\
+ \\
+ \\
0\end{array}$ & $\begin{array}{l}\text { VT } \\
\text { VF } \\
\text { VT (NS) } \\
\text { VT } \\
\text { VT } \\
\text { VT } \\
\text { VT } \\
\text { VT } \\
\text { VT } \\
\text { VT } \\
\text { VT } \\
\text { VT } \\
\text { VT } \\
\text { VT } \\
\text { VT } \\
\text { VT } \\
\text { VT } \\
\text { VT } \\
\text { VT } \\
\text { VT } \\
\text { VT } \\
\text { S } \\
\text { VF } \\
\text { VF } \\
\text { VF } \\
\text { VT } \\
\text { S } \\
\text { VT } \\
\text { VT } \\
\text { VT } \\
\text { VF } \\
\text { VT } \\
\text { VT }\end{array}$ & $\begin{array}{l}2 \\
2 \\
2 \\
2 \\
2 \\
2 \\
2 \\
2 \\
2 \\
2 \\
2 \\
2 \\
2 \\
2 \\
5 \\
5 \\
2 \\
5 \\
2 \\
5 \\
5 \\
2 \\
2 \\
2 \\
2 \\
5 \\
5 \\
5 \\
2 \\
2 \\
2 \\
2 \\
2 \\
2\end{array}$ & $\begin{array}{l}\text { SUVT } \\
\text { SUVT } \\
\text { SUVT } \\
\text { SUVT } \\
\text { SUVT } \\
\text { SUVT } \\
\text { SUVT } \\
\text { SUVT } \\
\text { SUVT } \\
\text { SUVT } \\
\text { SUVT } \\
\text { SUVT } \\
\text { SUVT } \\
\text { SUVT } \\
\text { SUVT } \\
\text { SUVT } \\
\text { SUVT } \\
\text { SUVT } \\
\text { SUVT } \\
\text { SUVT } \\
\text { SUVT } \\
\text { SUVT } \\
\text { VF } \\
\text { VF } \\
\text { VF } \\
\text { VF } \\
\text { VF } \\
\text { VF } \\
\text { VF } \\
\text { NUVT } \\
\text { NUVT } \\
\text { NUVT } \\
\text { NUVT } \\
\text { NUVT }\end{array}$ & $\begin{array}{l}\text { RVOT S2, S3 } \\
\text { RVA S2, S3 } \\
\text { RVA S1, S2 } \\
\text { RVA S2, S3 } \\
\text { RVA S2, S3 } \\
\text { RVA burst } \\
\text { RVA S2, S3 } \\
\text { RVA S1, S2 } \\
\text { RVA S2, S3 } \\
\text { RVA burst } \\
\text { RVA burst } \\
\text { RVA S1, S2 } \\
\text { RVA S2, S3 } \\
\text { RVA S1, S2 } \\
\text { RVA S1, S2 } \\
\text { RVA S1, S2 } \\
\text { RVA S2, S3 } \\
\text { RVA S1, S2 } \\
\text { RVA burst } \\
\text { RVA S1, S2 } \\
\text { RVA S2, S3 } \\
\text { RVA S2, S3 } \\
\text { RVA S2, S3 } \\
\text { RVA S2, S3 } \\
\text { RVOT S2, S3 } \\
\text { RVOT S2, S3 } \\
\text { RVA S2, S3 } \\
\text { RVA S2, S3 } \\
\text { RVA S2, S3 } \\
\text { RVA S1, S2 } \\
\text { RVA S2, S3 } \\
\text { RVA S2, S3 } \\
\text { RVA S2, S3 } \\
\text { RVA S1, S2 }\end{array}$ & $\begin{array}{l}2 \\
2 \\
2 \\
2 \\
2 \\
2 \\
2 \\
2 \\
2 \\
2 \\
2 \\
2 \\
2 \\
2 \\
5 \\
5 \\
5 \\
5 \\
5 \\
5 \\
5 \\
10 \\
2 \\
2 \\
2 \\
5 \\
5 \\
5 \\
5 \\
2 \\
2 \\
2 \\
2 \\
2\end{array}$ \\
\hline
\end{tabular}

CAD, coronary artery disease; IPC, initial pacing current; NUVT, non-sustained uniform ventricular tachycardia;

(NS), non-sustained; RVA, right ventricular apex; RVOT, right ventricular outflow tract; S, syncope; S1, S2, single extrastimulus; S2, S3, double extrastimuli; SUVT, sustained uniform ventricular tachycardia; VA, ventricular arrhythmia;

$\mathrm{VF}$, ventricular fibrillation; VT, documented ventricular tachycardia.

have a positive study if any of the above ventricular arrhythmias or ventricular fibrillation was induced.

\section{Results}

NEGATIVE STUDIES

No ventricular arrhythmias were induced in 36 patients. The negative studies occurred in $18(90 \%)$ of group 4 , four $(80 \%)$ of group 3, six $(55 \%)$ of group 2 , and eight $(23 \%)$ of group 1 . Six of the eight negative studies in group 1 were in patients whose original ventricular tachycardia had occurred in association with a predisposing clinical event (two during chest pain, one within 24 hours of cardiac surgery, one while on quinidine, one within three weeks of an infarct, and one during exercise). Five of the six negative studies in group 2 were in patients whose ventricular fibrillation had occurred under similar circumstances (two during chest pain, two within a week of infar ztion, and one within a week of coronary surgery). Thus the majority $(91 \%)$ of negative studies occurred in patients in whom no clinical arrhythmia had been identified or whose presenting arrhythmia was either non-sustained or had occurred in association with a recognised predisposing clinical event.

\section{POSITIVE STUDIES}

Ventricular arrhythmias were induced in 34 patients (table 2). Twenty two had sustained uniform ventricular tachycardia, seven had ventricular fibrillation, and five had non-sustained uniform ventricular tachycardia. In 16 of the patients in whom sustained uniform ventricular tachycardia was induced the arrhythmia was identical with the recorded clinical arrhythmia. In the remaining six patients we did not have 12 lead electrocardiograms of the clinical arrhythmia for comparison. No sustained multiform ventricular tachycardia was induced. Arrhythmias were induced in $26(77 \%)$ of group 1 , five $(45 \%)$ of group 2 , one $(20 \%)$ of group 3 , and two $(10 \%)$ oi group 4 . Thirty one of the arrhythmias were induced by stimulation at the right ventricular apex: 10 by a single extrastimulus, 17 by 


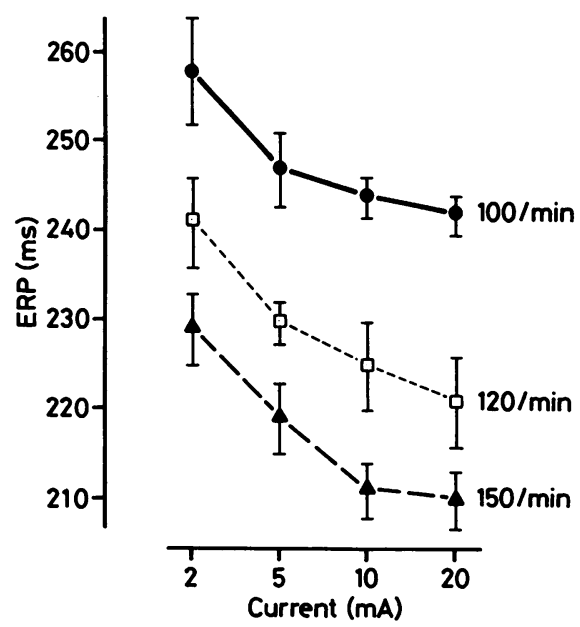

Figure The effects (mean (SEM)) of stimulation current on measured effective refractory period (ERP) during complete diastolic scans with a single extrastimulus in 47 patients at each basic pacing rate.

double extrastimuli, and four by burst pacing. The remaining three arrhythmias were induced by double extrastimuli at the right ventricular outflow tract.

\section{STIMULATION CURRENT}

The figure shows the effect of increasing stimulation current on measured effective refractory period at each basic pacing rate. This shows mean results from the 47 patients in whom diastolic scans were completed with a single extrastimulus at $2,5,10$, and 20 $\mathrm{mA}$ (that is, patients with a low pacing threshold and no arrhythmia induction with a single extrastimulus). Each increase in current caused a decrease in the measured effective refractory period. The increase in current from the initial stimulation current to $20 \mathrm{~mA}$ caused a mean (SEM) total decrease in effective refractory period of $19.0(2.0) \mathrm{ms}$ in those with a positive study and $17.6(3.0) \mathrm{ms}$ in those in whom the study was negative.

Ventricular arrhythmias were induced at $2 \mathrm{~mA}$ in 22 patients, at $5 \mathrm{~mA}$ in 11 , and at $10 \mathrm{~mA}$ in one. The increase from $10 \mathrm{~mA}$ to $20 \mathrm{~mA}$ caused only a small decrease in effective refractory period and did not induce any arrhythmias. Eight of the 12 patients whose arrhythmias were induced at a current greater than $2 \mathrm{~mA}$ had diastolic thresholds $>1 \mathrm{~mA}$ and were therefore paced at an initial current of $5 \mathrm{~mA}$. Thus in only four patients (cases 17, 19, 22, 29 in table 2) was it necessary to increase the pacing current above the initial level in order to induce an arrhythmia.

PATIENT FOLLOW UP

Most patients with a positive test have been recruited into a trial of antiarrhythmic drug treatment that required further electrophysiological studies which will be reported at a later date.

During a mean follow up of $15 \cdot 1(1-31)$ months in the patients in whom the test was negative, only one (a 16 year old boy with a cardiomyopathy) had a ventricular arrhythmia and required antiarrhythmic treatment. Two patients died, one of heart failure within 24 hours of coronary surgery. The other, a patient who had undergone surgery for severe coronary disease (including left main stenosis), died out of hospital 14 months after the study. When he was seen a month before death he had not experienced any arrhythmias but he was incapacitated by heart failure.

\section{Discussion}

To be of value a technique of programmed electrical stimulation should be sufficiently sensitive to identify those at risk of life threatening arrhythmias and sufficiently specific to exclude those who are not. The stimulation protocol should, therefore, induce clinically relevant arrhythmias in susceptible patients without inducing "artefactual" arrhythmias, such as multiform ventricular tachycardia and ventricular fibrillation, ${ }^{267}$ in others.

In our protocol we used only two extrastimuli since it has been shown that there is an increased risk of stimulating artefactual arrhythmias when three or more extrastimuli are used. ${ }^{8-10}$ We specifically examined the effects of increasing stimulation current on ventricular refractoriness and arrhythmia induction because of the continuing controversy over the best stimulation current for such protocols.

It has been shown that ventricular refractoriness varies greatly between areas of normal and diseased myocardium and between closely adjacent areas of damaged myocardium. ${ }^{11}$ In damaged areas the ventricular refractory period can often be so prolonged that currents greater than twice the diastolic threshold are needed to allow sufficiently early ventricular capture for an arrhythmia to be induced. Under these circumstances the pacing threshold is not a good indicator of ventricular refractoriness. There is, therefore, no means of knowing whether a pacing electrode is adjacent to normal or abnormal myocardium at the start of a stimulation protocol. This heterogeneity of ventricular refractoriness argues against the exclusive use of low stimulation currents (conventionally set at twice the diastolic threshold) in programmed electrical stimulation. On the other hand, the prolonged use of high stimulation currents probably has arrhythmogenic effects independent of its effects on ventricular refractoriness. ${ }^{1412}$ 
Richards et al used both low (twice diastolic threshold) and high $(20 \mathrm{~mA})$ stimulation currents with a maximum of two extrastimuli to study 165 patients within 28 days of an acute myocardial infarction. ${ }^{3}$ The exclusive use of low stimulation currents in their protocol would have failed to identify four patients at risk of subsequent ventricular arrhythmias. They conceded, however, that high current stimulation also may have produced an unknown number of false positive results. Morady et $a l$, studying a population similar to our own, found that a stimulation current of $10 \mathrm{~mA}$ induced ventricular arrhythmias in $\mathbf{1 6}$ of 26 patients in whom no arrhythmias had been induced at twice the diastolic threshold. ${ }^{4}$ The clinical relevance of these arrhythmias was doubtful, however, and they concluded that a high current may compromise the specificity of a protocol to a greater degree than it enhances its sensitivity. A similar conclusion was reached by Kennedy et al who compared the use of high and low stimulation currents with up to three extrastimuli in 15 patients studied for indications other than sustained ventricular tachycardia. ${ }^{5}$ In this study, increasing the current from twice the diastolic threshold to $10 \mathrm{~mA}$ induced six episodes of ventricular fibrillation, only one of which was thought to be clinically relevant. Herre et al also reported a high incidence of non-clinical arrhythmias when a stimulation current of $10 \mathrm{~mA}$ and up to four extrastimuli were used. ${ }^{1}$ In most of the above instances the increased arrhythmogenicity of high stimulation current was attributed to earlier capture of the ventricle than was possible at lower currents. Both Herre et al and Morady et al, however, found that arrhythmias were induced by high stimulation currents at coupling intervals that had failed to induce an arrhythmia at lower currents. ${ }^{14}$ This suggests that a high stimulation current has an independent arrhythmogenic effect in addition to its effects on ventricular refractoriness.

In our study the stimulation current was incrementally increased only after lower currents had failed to capture the ventricle. The effect of increasing current in this manner is simply to facilitate earlier capture of the ventricle than is possible at lower currents. This approach avoids the prolonged use of high stimulation currents, yet fully exploits the current-interval properties of the area of ventricle adjacent to the electrode. ${ }^{13}$

The majority of arrhythmias in our patients were induced at the lowest current used (22 at $2 \mathrm{~mA}$ and eight at $5 \mathrm{~mA}$ ). In the remaining four the increase in current induced an arrhythmia by allowing earlier capture of the ventricle than was possible at the initial current. Although the increase from 5 to $10 \mathrm{~mA}$ caused a significant decrease in mean ventricular refractoriness it resulted in only one clinically relevant arrhythmia, while the increase from 10 to 20 $\mathrm{mA}$ had little effect on ventricular refractoriness and induced no arrhythmias. Thus a single stimulation current of $5 \mathrm{~mA}$ would have identified all but one of our patients at risk of further ventricular arrhythmias. This allows simplification of the protocol for routine clinical use since our results, and most of those cited above, clearly show that there is little to be gained by using currents of $10 \mathrm{~mA}$ and above. Indeed this would only serve to increase the risk of inducing artefactual arrhythmias. ${ }^{6}$

Our follow up shows that the protocol was sensitive enough to detect all but one of our population at risk of recurrent ventricular arrhythmias. Specificity is more difficult to assess, however. Since we studied several patients in whom the likelihood of inducing an arrhythmia was small (group 4), the low incidence $(10 \%)$ of induced arrhythmias in this group is encouraging. Indeed in our study programmed electrical stimulation was of limited value in patients in whom no clinical arrhythmia had been identified; this probably reflects the high proportion $(60 \%)$ of patients without structural heart disease in this group.

We induced no multiform ventricular tachycardia in patients who did not go on to have a sustained arrhythmia and we induced ventricular fibrillation in only seven patients. The clinical importance of induced ventricular fibrillation is uncertain since it can be induced in most normal hearts by a sufficiently aggressive protocol. ${ }^{6}$ Spielman et al suggested that its induction was indicative of an increased risk of subsequent ventricular arrhythmias in patients who had presented with a clinical ventricular arrhythmia. ${ }^{14}$ By contrast, DiCarlo et al concluded that it was a non-specific finding in patients who had not previously had sustained ventricular arrhythmias. ${ }^{7}$ Several investigators have shown that the incidence of ventricular fibrillation is increased by using high stimulation currents. ${ }^{4-6}$

Five of our seven patients with induced ventricular fibrillation had presented with a sustained ventricular arrhythmia (three with ventricular tachycardia and two with ventricular fibrillation) and six of the episodes were induced at the lowest current used. We therefore feel that these are unlikely to be artefactual responses, although we recognise the need for caution in the interpretation of this response. The same is probably true of the five episodes of non-sustained uniform ventricular tachycardia. Each occurred at the lowest stimulation current in patients who had presented with sustained ventricular arrhythmias. While the clinical relevance of this repetitive response is uncertain, it has been shown to predict a poor prognosis when it is induced 
after antiarrhythmic treatment. ${ }^{15}$

We have assessed an electrophysiological protocol for the investigation of patients thought to be susceptible to serious ventricular arrhythmias and have prospectively evaluated its use in 70 consecutive patients. The protocol was found to be highly sensitive and induced few, if any, artefactual arrhythmias. We have shown that the use of high stimulation currents is unnecessary and that a simplified protocol of two extrastimuli and a single stimulation current of $5 \mathrm{~mA}$ should identify the majority of patients whose symptoms are caused by recurrent ventricular arrhythmias.

PLW was in receipt of a Medical Research Council travelling fellowship.

\section{References}

1 Herre JM, Mann DE, Luck JC, et al. Effect of increased current, multiple pacing sites and number of extrastimuli on induction of ventricular tachycardia. Am J Cardiol 1986;57:102-7.

2 Wellens HJJ, Brugada P, Stevenson WG. Programmed electrical stimulation of the heart in patients with life-threatening ventricular arrhythmias: what is the significance of induced arrhythmias and what is the correct stimulation protocol? Circulation 1985; 72:1-7.

3 Richards DA, Cody DV, Denniss AR, Russell PA, Young AA, Uther JB. Ventricular electrical instability: a predictor of death after myocardial infarction. Am J Cardiol 1983;51:75-80.

4 Morady F, DiCarlo LA, Bing Liem L, Krol RB, Baerman JM, Peter T. Effects of high stimulation current on the induction of ventricular tachycardia. Am J Cardiol 1985;56:73-8.

5 Kennedy EE, Rosenfeld LE, McPherson CA, Stark SI, Batsford WP. Mechanisms and relevance of arrhythmias induced by high-current programmed ventricular stimulation. Am J Cardiol 1986;57:598-603.
6 Hamer AW, Karagueuzian HS, Sugi K, Zaher CA, Mandel WJ, Peter T. Factors related to the induction of ventricular fibrillation in the normal canine heart by programmed electrical stimulation. J Am Coll Cardiol 1984;3:751-9.

7 DiCarlo AL, Morady F, Schwartz AB, et al. Clinical significance of ventricular fibrillation-flutter induced by ventricular programmed stimulation. Am Heart $J$ 1985;109:959-63.

8 Brugada $\mathrm{P}$, Abdollah $\mathrm{H}$, Heddle B, Wellens $\mathrm{HJJ}$. Results of a ventricular stimulation protocol using a maximum of 4 premature stimuli in patients without documented or suspected ventricular arrhythmias. Am J Cardiol 1983;52:1214-8.

9 Mann DE, Luck JC, Griffin JC, et al. Induction of ventricular tachycardia using programmed stimulation: value of third and fourth extrastimuli. Am J Cardiol 1983;52:501-6.

10 Buxton AE, Waxman HL, Marchlinski FE, Untereker WJ, Waspe LE, Josephson ME. Role of triple extrastimuli during electrophysiologic study of patients with documented sustained ventricular tachyarrhythmias. Circulation 1984;69:532-40.

11 Michelson EL, Spear JF, Moore EN. Strength-interval relations in a chronic canine model of myocardial infarction. Implications for the interpretation of electrophysiologic studies. Circulation 1981;63:1158-65.

12 Spear JF, Moore EN, Horowitz LN. Effect of current pulse delivered during the ventricular vulnerable period upon the ventricular fibrillation threshold. Am J Cardiol 1973;32:814-22.

13 Camardo JS, Greenspan AM, Horowitz LN, Spielman SR, Josephson ME. Strength-interval relation in the human ventricle: effect of procainamide. $\mathrm{Am} \mathrm{J} \mathrm{Car-}$ diol 1980;45:856-60.

14 Spielman SR, Farshidi A, Horowitz LN, Josephson ME. Ventricular fibrillation during programmed ventricular stimulation: incidence and clinical implications. Am J Cardiol 1978;42:913-8.

15 Platia EV, Reid PR. Nonsustained ventricular tachycardia during programmed ventricular stimulation: criteria for a positive test. Am J Cardiol 1985; 56:79-83. 